\title{
Low-complexity trellis decoding of linear block codes
}

\author{
B. Honary \\ G. Markarian \\ M. Darnell
}

Indexing terms: Trellis decoding, Linear block codes

\begin{abstract}
The authors introduce a new simple encoding technique which allows the design of a wide variety of linear block codes. They present a number of examples in which the most widely used codes (Reed-Muller, Hamming, Golay, optimum etc.) have been designed. They also introduce a novel trellis design procedure for the proposed codes. It is shown that the trellises of the designed codes are similar to the trellises of coset codes and allow low complexity soft maximum likelihood decoding.
\end{abstract}

\section{Introduction}

Trellis decoding technique for linear block codes has been under investigation since 1974 [1-3]. Later, a number of efficient soft decision maximum likelihood decoding (MLD) algorithms for block codes were proposed $[4,6,7]$. Recent publications by Forney $[8,9]$ and others $[5,10,11]$ have stimulated interest in lowcomplexity trellis decoding of block codes for both practical and theoretical reasons. On the practical side, a minimal trellis structure of a block code can be used for performing low-complexity maximum likelihood soft decision decoding. From the theoretical viewpoint, such trellises can be used for construction of other codes [10].

Recently, generalised array codes (GACs) and their trellis structures have been introduced [12]. The technique allows the design of array codes with the same code length, $n$, and $d_{\text {min }}=4$, but with an increased number of information digits. These codes are simple and flexible to design; they also allow low-complexity soft maximum likelihood trellis decoding (SMLTD). It has been shown that the concept of GACs can be applied to the design of Hamming, Reed-Muller (RM) and Golay codes with a low-complexity trellis structure $[13,14]$. In this paper we describe a new encoding/decoding technique for different types of linear codes based on GACs and their trellis structure. The trellises of the designed codes are similar to trellises of the coset codes designed by Forney [8] and provide lower complexity SMLTD than that achievable with conventional techniques.

(C) IEE, 1995

Paper 2037I (E5), first received 2nd September 1994 and in revised form 24th April 1995

B. Honary and G. Markarian are with the Communications Research Centre, Lancaster University, Lancaster LA1 4YR, United Kingdom

M. Darnell is with the Department of Electronic and Electrical Engineering, University of Leeds, Leeds LS2 9JT, United Kingdom

\section{Code construction}

A generalised $\left(n, k, d_{\min }\right)$ array code is an array code in which the column and row subcodes may have different numbers of information and parity check symbols; the code length $n=n_{1} n_{2}$ and the total number of information digits $k=k_{1}+k_{2}+\cdots+k_{n_{2}}$, where $n_{1}$ and $n_{2}$ represent the number of columns and rows respectively, $k_{p}$ is the number of information digits in the $p$ th row [12]. Using the concept of the GACs, a wide variety of known linear block codes together with their low complexity trelisses can be designed. The procedure for designing a linear $\left(n_{0}, k_{0}, d_{0}\right) \mathrm{GAC}$ is as follows:

(i) Design a binary, $n=n_{1} n_{2},\left(n=n_{0}\right)$ basic product code, $C_{1}$, as shown in Fig. $1 a$, with a single parity check column and $R_{1}=\left(n_{1}, k_{1}, d_{1}\right)$ row codes, where $d_{1}=$ $\left\lceil d_{0} / 2\right\rceil$ and $\left.\Gamma \cdot\right\rceil$ is the nearest greatest integer (if $n_{0}$ is a prime number choose $n=n_{0}+1$ ).

(ii) Design a binary, $n_{1} n_{2}$, additional product code, $C_{2}=|P A|$, as shown in Fig. $1 b$, where $P$ is a binary $k_{1} n_{2}$, array with only parity check elements; $A$ is a binary, $\left(n_{1}-k_{1}\right) n_{2}$, matrix where the first row consists of only $k^{\prime}=n_{1}-k_{1}$ information digits, and all column codes are repetition codes.

(iii) Design (if not all information digits have been used) a second additional binary product code, $C_{3}$, as shown in Fig. $1 c$, where $B=\left(n_{2}, 1, d_{0}\right)$ is a repetition row code with $k_{0}$ th information digit.

(iv) Add the designed codes as follows:

$C=C_{1} \oplus C_{2} \oplus C_{3}$

where addition is on modulo -2 basis

(iv) If $n=n_{0}+1$, delete the symbol which is located in the $n_{2}$ th row and $n_{1}$ th column.

This procedure can be also described as an array representation of coset codes introduced by Forney [8]. Code $C_{1}$, is a linear product codes, and the overall designed $C$ can be represented as a union of cosets of $C_{1}$. This union forms a linear nonsystematic code with the following parameters: $\left(n_{0}, k_{0}, d_{0}\right)$. In this section we show that the proposed technique can be employed for the design of a wide variety of linear block codes.
The authors would like to express their gratitude towards Prof. R.J. McEliece for his very helpful advice and comments regarding the revision of the paper. This work was supported by the Science and Engineering Research Council (SERC), UK. 
$2.7(8,4,4)$ and $(7,4,3)$ codes design

An example is given for an $(8,4,4)$ GAC which is equivalent to the well known $(8,4,4)$ RM code $[15,16]$. The procedure for code design is given below:

(i) Design the basic, $(8,3,4),\left(n=2 \times 4, n_{1}=2, n_{2}=4\right)$ array code, $C_{1}$, with the single-parity check $(4,3,2)$ column and repetition $R_{1}=(2,1,2)$ row codes $\left(d_{1}=\right.$ $d_{0} / 2=2$ ):

$$
C_{1}=\left[\begin{array}{ll}
x_{1} & p_{1} \\
x_{2} & p_{2} \\
x_{3} & p_{3} \\
p_{4} & p_{4}
\end{array}\right]
$$
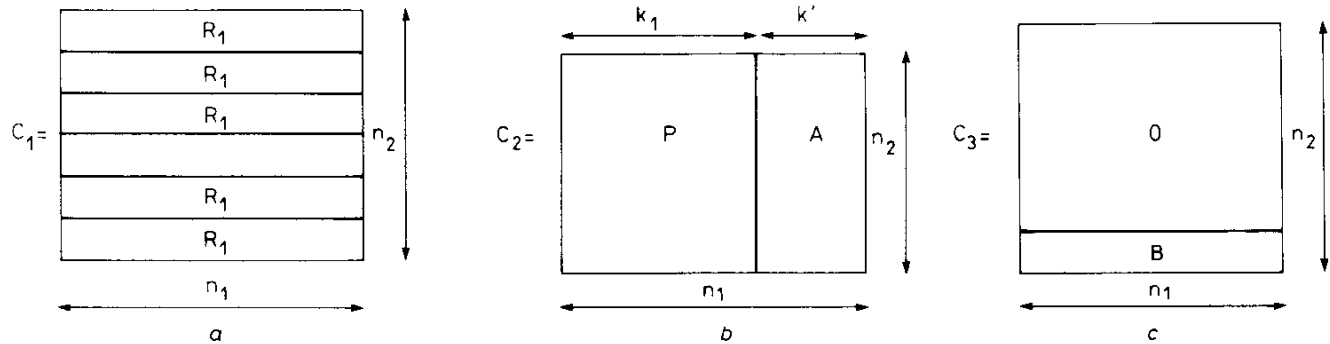

Fig. 1 Code structures

$a$ Basic product code, $\mathrm{C}_{1} \quad b$ Additional product code, $\mathrm{C}_{2} \quad c$ Additional product code, $\mathrm{C}_{3}$

where $x_{i}, i=1,2,3$ represent information digits and $p_{j}$, $j=1, \ldots, 4$, represent parity check symbols:

$$
\left.\begin{array}{l}
p_{j}=x_{j} \forall j \leqslant 3 \\
p_{4}=x_{1}+x_{2}+x_{3}
\end{array}\right\}
$$

(ii) Design an additional array code, $C_{2}=|P A|$ with the following structure:

$$
C_{2}=\left[\begin{array}{ll}
0 & x_{4} \\
0 & x_{4} \\
0 & x_{4} \\
0 & x_{4}
\end{array}\right]
$$

where $x_{4}$ is an information digit, and $\mathrm{P}$ is an all-zero column.

(iii) Since all information digits have been used, there is no need to design a second additional array code, $C_{3}$.

(iv) Add the two codes $C_{1}$ and $C_{2}$ on a modulo-2 basis and read the designed code row-by-row as follows:

$$
\begin{aligned}
C & =C_{1} \oplus C_{2}=\left[\begin{array}{ll}
x_{1} & x_{4} \oplus p_{1} \\
x_{2} & x_{4} \oplus p_{2} \\
x_{3} & x_{4} \oplus p_{3} \\
p_{4} & x_{4} \oplus p_{4}
\end{array}\right] \\
& =\left(x_{1}, x_{4} \oplus p_{1}, x_{2}, x_{4} \oplus p_{2}, x_{3}, x_{4} \oplus p_{3}, p_{4}, x_{4} \oplus p_{4}\right)
\end{aligned}
$$

(v) Since $n_{0}=n$, there is no need for the deletion of the parity-check symbol which is located in the 4th row and 2nd column.

The designed code is a linear nonsystematic code and has the following parameters: $\left(n_{0}=8, k_{0}=4, d_{0}=4\right)$. The weight distribution function (WDF) of the code is shown in Fig. 2 and is similar to the WDF of the $(8,4,4)$ RM code. It has been shown [13] that by deleting the symbol which is located in the second column and 4th row, the designed $(8,4,4)$ code will be transformed to the $(7,4,3)$ Hamming code:

$$
\begin{aligned}
C & =C_{1} \oplus C_{2}=\left[\begin{array}{ll}
x_{1} & x_{4} \oplus p_{1} \\
x_{2} & x_{4} \oplus p_{2} \\
x_{3} & x_{4} \oplus p_{3} \\
p_{4} &
\end{array}\right] \\
& =\left(x_{1}, x_{4} \oplus p_{1}, x_{2}, x_{4} \oplus p_{2}, x_{3}, x_{4} \oplus p_{3}, p_{4}\right)
\end{aligned}
$$

\section{$2.2(16,5,8)$ and $(15,57)$ codes design}

An example is now given for $(16,5,8)$ and $(15,5,7)$

GACs. The procedure for codes design is shown below:

(i) Design the basic, $(16,3,8),\left(n=4 \times 4, n_{1}=n_{2}=4\right)$ product code, $C_{1}$, with the repetition row code $R_{1}=(4,1$, $4)$, and $(4,3,2)$ single parity check column code $\left(d_{1}=\right.$ $\left.d_{0} / 2=4\right)$ :

$$
C_{1}=\left[\begin{array}{llll}
x_{1} & p_{1} & p_{1} & p_{1} \\
x_{2} & p_{2} & p_{2} & p_{2} \\
x_{3} & p_{3} & p_{3} & p_{3} \\
p_{4} & p_{4} & p_{4} & p_{4}
\end{array}\right]
$$

where $x_{i}, i=1,2,3$, represent information digits and parity check symbols, $p_{j}, j=1,2,3,4$, are defined according to eqn. 3 .

(ii) Design an additional product code, $C_{2}=|P A|$ with the following structure:

$$
C_{2}=\left[\begin{array}{llll}
0 & p_{5} & x_{4} & x_{5} \\
0 & p_{5} & x_{4} & x_{5} \\
0 & p_{5} & x_{4} & x_{5} \\
0 & p_{5} & x_{4} & x_{5}
\end{array}\right]
$$

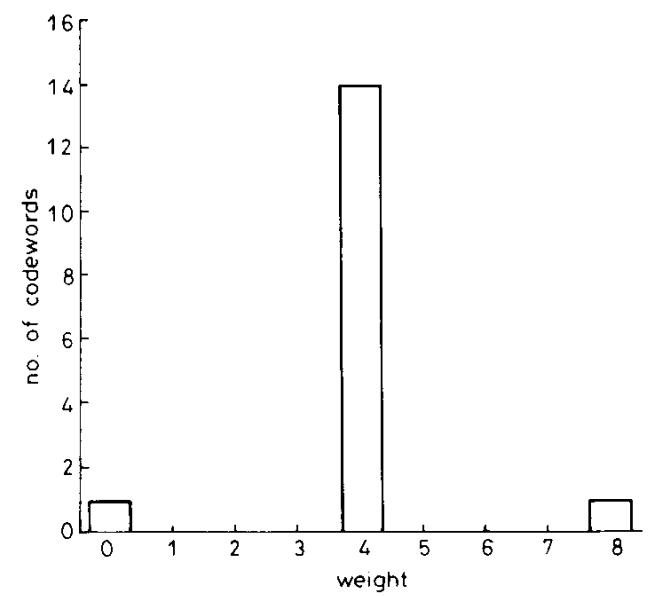

Fig. 2 Weight distribution function of $(8,4,4) G A C$ 
where $x_{4}$ and $x_{5}$ are information digits, $P$ is a $2 \times 4$ parity matrix and $p_{5}=x_{4}+x_{5}$

(iii) Since all information digits have been used, there is no need to design a second additional array code, $C_{3}$.

(iv) Add the two codes $C_{1}$ and $C_{2}$ on a modulo 2 basis as follows:

$$
C=C_{1} \oplus C_{2}=\left[\begin{array}{llll}
x_{1} & p_{5} \oplus p_{1} & x_{4} \oplus p_{1} & x_{5} \oplus p_{1} \\
x_{2} & p_{5} \oplus p_{2} & x_{4} \oplus p_{2} & x_{5} \oplus p_{2} \\
x_{3} & p_{5} \oplus p_{3} & x_{4} \oplus p_{3} & x_{5} \oplus p_{3} \\
p_{4} & p_{5} \oplus p_{4} & x_{4} \oplus p_{4} & x_{5} \oplus p_{4}
\end{array}\right]
$$

This code is equivalent to the $(16,5,8) \mathrm{RM}$ code from which a $(15,5,7)$ GAC can be derived by simple deletion of the parity chack symbol located in the 4th row and 4th column:

$$
C=\left[\begin{array}{llll}
x_{1} & p_{5} \oplus p_{1} & x_{4} \oplus p_{1} & x_{5} \oplus p_{1} \\
x_{2} & p_{5} \oplus p_{2} & x_{4} \oplus p_{2} & x_{5} \oplus p_{2} \\
x_{3} & p_{5} \oplus p_{3} & x_{4} \oplus p_{3} & x_{5} \oplus p_{3} \\
p_{4} & p_{5} \oplus p_{4} & x_{4} \oplus p_{4} &
\end{array}\right]
$$

The weight distribution function (WDF) of the designed code is shown in Fig. 3. It is evident that the derived code has the following parameters: $\left(n_{0}=15, k_{0}=5, d_{0}=7\right)$, and is equivalent to a corresponding $\mathrm{BCH}$ code.

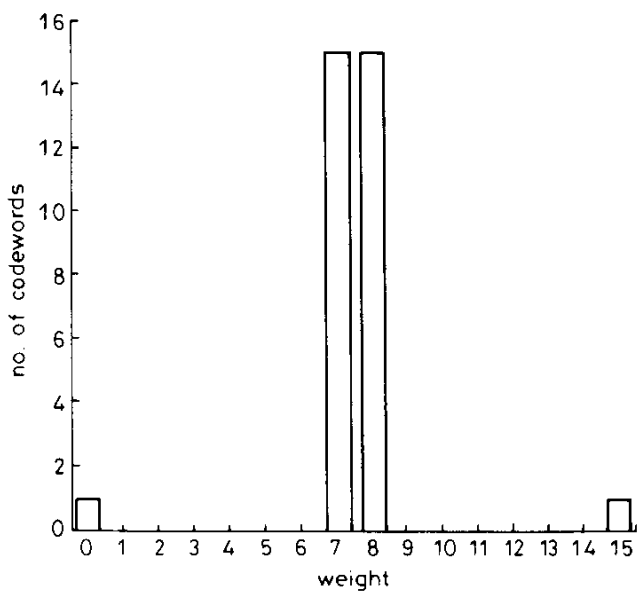

Fig. 3 Weight distribution function of $(15,5,7) G A C$

\section{$2.3(16,11,4)$ and $(15,11,3)$ codes design}

The proposed technique can be implemented for the design of other RM codes with higher information rates. An example is given for a $(16,11,4)$ GAC which is equivalent to the corresponding RM code. The procedure for code design is as follows:

(i) Design a basic $4 \times 4$ array code $C_{1}$, with the single parity check row and column codes $\left(R_{1}=(4,3,2), d_{1}=\right.$ $\left.d_{0} / 2\right)$ :

$$
C_{1}=\left[\begin{array}{llll}
x_{1} & x_{2} & x_{3} & p_{1} \\
x_{4} & x_{5} & x_{6} & p_{2} \\
x_{7} & x_{8} & x_{9} & p_{3} \\
p_{4} & p_{5} & p_{6} & p_{7}
\end{array}\right]
$$

where $x_{i}, i=1,2, \ldots, 9$, represent information digits and $p_{j}, j=1,2, \ldots, 7$, represent single parity check digits for row and column codes. $|P A|$

(ii) Design a binary additional product code $C_{2}=$

$$
C_{2}=\left[\begin{array}{llll}
0 & 0 & 0 & x_{10} \\
0 & 0 & 0 & x_{10} \\
0 & 0 & 0 & x_{10} \\
0 & 0 & 0 & x_{10}
\end{array}\right]
$$

where $P$ is an all zero $4 \times 3$ matrix, $A$ is a $4 \times 1$ repetition column matrix and $x_{10}$ is an information digit.

(iii) Since not all information digits have been used, design second additional array, $C_{3}$, with the repetition code at the last row

$$
C_{3}=\left[\begin{array}{cccc}
0 & 0 & 0 & 0 \\
0 & 0 & 0 & 0 \\
0 & 0 & 0 & 0 \\
x_{11} & x_{11} & x_{11} & x_{11}
\end{array}\right]
$$

where $x_{11}$ is an information digit.

(iv) Add the three codes on a modulo-2 basis as follows:

$$
\begin{aligned}
C & =C_{1} \oplus C_{2} \oplus C_{3} \\
& =\left[\begin{array}{cccc}
x_{1} & x_{2} & x_{3} & p_{1} \oplus x_{10} \\
x_{4} & x_{5} & x_{6} & p_{2} \oplus x_{10} \\
x_{7} & x_{8} & x_{9} & p_{3} \oplus x_{10} \\
p_{4} \oplus x_{11} & p_{5} \oplus x_{11} & p_{6} \oplus x_{11} & p_{7} \oplus x_{11} \oplus x_{10}
\end{array}\right]
\end{aligned}
$$

It follows from the construction that the designed code is a $(16,11,4) \mathrm{GAC}$ which is equivalent to the corresponding $\mathrm{RM}$ code. It is also apparent that the $(15,11,3)$ Hamming code can be simply derived by deleting the parity check symbol located in the 4th row and 4th column of the designed $(16,11,4)$ code.

\section{$2.4(15,7,5)$ code design}

The procedure outlined above can be applied to the design of a $(15,7,5) \mathrm{GAC}$. To illustrate the flexibility of the proposed technique, we choose a different size of the basic array. The encoding procedure is as follows:

(i) Design the basic, $(15,4,6)\left(n=5 \times 3, n_{1}=5, n_{2}=\right.$ 3) product code, $C_{1}$, with a $R_{1}=(5,2,3)$ row code $\left(d_{1}=\right.$ $\left.\left[d_{0} / 2\right]=3\right)$ and $(3,2,2)$ single parity check column code:

$$
C_{1}=\left[\begin{array}{lllll}
x_{1} & x_{2} & p_{1} & p_{2} & p_{3} \\
x_{3} & x_{4} & p_{4} & p_{5} & p_{6} \\
p_{7} & p_{8} & p_{9} & p_{10} & p_{11}
\end{array}\right]
$$

where $x_{i}, i=1,2,3,4$, represent information digits and $p_{j}, j=1, \ldots, 11$, represent parity check symbols.

(ii) Design two additional product codes, $C_{2}$ and $C_{3}$ with the following structure:

$$
\begin{aligned}
C_{2} & =\left[\begin{array}{lllll}
0 & 0 & p_{12} & x_{5} & x_{6} \\
0 & 0 & p_{12} & x_{5} & x_{6} \\
0 & 0 & p_{12} & x_{5} & x_{6}
\end{array}\right] \\
C_{3} & =\left[\begin{array}{ccccc}
0 & 0 & 0 & 0 & 0 \\
0 & 0 & 0 & 0 & 0 \\
x_{7} & x_{7} & x_{7} & x_{7} & x_{7}
\end{array}\right]
\end{aligned}
$$

where $x_{i}, i=5,6,7$ represent information digits and $p_{12}=x_{5}+x_{6}$. 
(iii) Add the three codes on a modulo-2 basis, as follows:

$$
\begin{aligned}
C= & C_{1} \oplus C_{2} \oplus C_{3} \\
= & {\left[\begin{array}{ccc}
x_{1} & x_{2} & p_{1} \oplus p_{12} \\
x_{3} & x_{4} & p_{4} \oplus p_{12} \\
p_{7} \oplus x_{7} & p_{8} \oplus x_{7} & p_{9} \oplus x_{7} \oplus p_{12} \\
& x_{5} \oplus p_{2} & x_{6} \oplus p_{3} \\
x_{5} \oplus p_{5} & x_{6} \oplus p_{6} \\
p_{10} \oplus x_{7} \oplus x_{5} & p_{11} \oplus x_{7} \oplus x_{6}
\end{array}\right] }
\end{aligned}
$$

The designed code has the following parameters: $\left(n_{0}=\right.$ $15, k_{0}=7, d_{0}=5$ ), and is equivalent to a corresponding BCH code.

\section{$2.5(17,9,5)$ code design}

Application of the proposed technique is not restricted to the RM and Hamming codes. The technique provides a 'good' co-ordinate reordering for other codes, for example optimum codes. An example is given for $(17,9$, 5) optimum code [16]; however, other known optimum codes can be obtained easily. The procedure is as follows:

(i) Design the basic $(18,6,6)\left(n=6 \times 3, n_{1}=6, n_{2}=3\right.$, $\left.n=n_{0}+1\right)$ product code, $C_{1}$, with a $R_{1}=(6,3,3)$ row code $\left(d_{1}=\left\lceil d_{0} / 2\right\rceil=3\right)$, and $(3,2,2)$ single parity check column code:

$$
C_{1}=\left[\begin{array}{llllll}
x_{1} & x_{2} & x_{3} & p_{1} & p_{2} & p_{3} \\
x_{4} & x_{5} & x_{6} & p_{4} & p_{5} & p_{6} \\
p_{7} & p_{8} & p_{9} & p_{10} & p_{11} & p_{12}
\end{array}\right]
$$

where $x_{i}, i=1,2, \ldots, 6$, represent information digits and $p_{j}, j=1, \ldots, 12$, represent parity check symbols.

(ii) Design two additional product codes, $C_{2}$ and $C_{3}$, as follows:

$$
\begin{aligned}
C_{2} & =\left[\begin{array}{llllll}
0 & 0 & 0 & p_{13} & x_{7} & x_{8} \\
0 & 0 & 0 & p_{13} & x_{7} & x_{8} \\
0 & 0 & 0 & p_{13} & x_{7} & x_{8}
\end{array}\right] \\
C_{3} & =\left[\begin{array}{cccccc}
0 & 0 & 0 & 0 & 0 & 0 \\
0 & 0 & 0 & 0 & 0 & 0 \\
x_{9} & x_{9} & x_{9} & x_{9} & x_{9} & x_{9}
\end{array}\right]
\end{aligned}
$$

$C=\left[\begin{array}{ccc}x_{1} \oplus c_{1} & x_{2} \oplus c_{2} & x_{3} \oplus c_{3} \\ x_{5} \oplus c_{1} & x_{6} \oplus c_{2} & x_{7} \oplus c_{3} \\ x_{12} \oplus p_{9} \oplus c_{1} & x_{12} \oplus p_{10} \oplus c_{2} & x_{12} \oplus p_{11} \oplus c_{3}\end{array}\right.$

$$
\left.\begin{array}{cccc}
x_{3} \oplus c_{3} & x_{4} \oplus c_{4} & & \\
x_{7} \oplus c_{3} & x_{8} \oplus c_{4} & & \\
x_{12} \oplus p_{11} \oplus c_{3} & x_{12} \oplus p_{12} \oplus c_{4} & & \\
p_{1} \oplus c_{5} & p_{2} \oplus x_{9} & p_{3} \oplus x_{10} & p_{4} \oplus x_{11} \\
p_{5} \oplus c_{5} & p_{6} \oplus x_{9} & p_{7} \oplus x_{10} & p_{8} \oplus x_{11} \\
x_{12} \oplus p_{13} \oplus c_{5} & x_{12} \oplus p_{14} \oplus x_{9} & x_{12} \oplus p_{15} \oplus x_{10} & x_{12} \oplus p_{16} \oplus x_{11}
\end{array}\right]
$$

where $x_{i}, i=7,8,9$, represent information digits and $p_{13}=x_{7}+x_{8}$.

(iii) Add the three codes, $C_{1}, C_{2}$ and $C_{3}$, on a modulo2 basis;

(iv) Delete the symbol which is located in the 3rd row and 6th column:

$$
\begin{aligned}
C= & {\left[\begin{array}{cccc}
x_{1} & x_{2} & x_{3} & \\
x_{4} & x_{5} & x_{6} & \\
p_{7} \oplus x_{9} & p_{8} \oplus x_{9} & p_{9} \oplus x_{9} & \\
p_{1} \oplus p_{13} & p_{2} \oplus x_{7} & p_{3} \oplus x_{8} \\
p_{4} \oplus p_{13} & p_{5} \oplus x_{7} & p_{6} \oplus x_{8} \\
p_{10} \oplus p_{13} \oplus x_{9} & p_{11} \oplus x_{7} \oplus x_{9} &
\end{array}\right] }
\end{aligned}
$$

It is apparent that the designed code is a linear nonsystematic optimum code with the following parameters: $\left(n_{0}=17, k_{0}=9, d_{0}=5\right)$.

\subsection{Golay codes design}

The $(24,12,8)$ binary extended Golay code occupies a remarkable place among binary block codes [8]. It is a unique, perfect, self-dual code which has been intensively investigated with various efficient encoding and decoding algorithms $[4,6,17]$. The trellis diagram for the $(24,12$, 8 ) extends Golay code, which consists of 64 states, has been introduced by Forney [8]. However, this technique requires complex encoder and can not be used for the trellis design of the $(23,12,7)$ Golay code. In this section we implement the concept of GACs for the design of both $(24,12,8)$ and $(23,12,7)$ Golay codes. We start with the design of $(24,12,8)$ extended Golay code. The procedure is as follows:

(i) Design the basic $(24,8,8)\left(n=8 \times 3, n_{1}=8, n_{2}=\right.$ 3 ) product code, $C_{1}$, with the $(3,2,2)$ column and $(8,4,4)$ row codes:

$$
C_{1}=\left[\begin{array}{cccccccc}
x_{1} & x_{2} & x_{3} & x_{4} & p_{1} & p_{2} & p_{3} & p_{4} \\
x_{5} & x_{6} & x_{7} & x_{8} & p_{5} & p_{6} & p_{7} & p_{8} \\
p_{9} & p_{10} & p_{11} & p_{12} & p_{13} & p_{14} & p_{15} & p_{16}
\end{array}\right]
$$

where $x_{i}, i=1,2, \ldots, 8$, represent information digits; $p_{j}$, $j=1, \ldots, 16$, represent parity check symbols and the $(8,4,4)$ row code has been described in Section 2.1 .

(ii) Design two additional product codes, $C_{2}$ and $C_{3}$, with the following structures:

$$
\begin{aligned}
C_{2} & =\left[\begin{array}{llllllll}
c_{1} & c_{2} & c_{3} & c_{4} & c_{5} & x_{9} & x_{10} & x_{11} \\
c_{1} & c_{2} & c_{3} & c_{4} & c_{5} & x_{9} & x_{10} & x_{11} \\
c_{1} & c_{2} & c_{3} & c_{4} & c_{5} & x_{9} & x_{10} & x_{11}
\end{array}\right] \\
C_{3} & =\left[\begin{array}{cccccccc}
0 & 0 & 0 & 0 & 0 & 0 & 0 & 0 \\
0 & 0 & 0 & 0 & 0 & 0 & 0 & 0 \\
x_{12} & x_{12} & x_{12} & x_{12} & x_{12} & x_{12} & x_{12} & x_{12}
\end{array}\right]
\end{aligned}
$$

where row code in $C_{2}$ is $(8,3,3)$ code and is shown in Table 1.

(iii) Add the three codes, $C_{1}, C_{2}$ and $C_{3}$ on a modulo-2 basis:

The weight distribution function for the designed $(24,12$, 8 ) code is shown in Fig. $4 a$. This is similar to the WDF of the $(24,12,8)$ extended Golay code [17], thus, following Reference 17, the designed code is an extended $(24,12,8)$ Golay code. The $(23,12,7)$ code can be derived by simple deletion of the parity-check symbol which is located in the 3rd row and 8th column. The weight distribution function of the designed $(23,12,7)$ code is shown in Fig. $4 b$. This is identical to the weight distribution function of the $(23,12,7)$ Golay code [17]

Example: A binary vector of information digits is given as: $X=\left(x_{1}, x_{2}, \ldots, x_{12}\right)=(001101011111)$. Following the procedure outlined above, $C_{1}, C_{2}$ and $C_{3}$ codewords 
can be written as follows:

$$
\begin{aligned}
C_{1} & =\left[\begin{array}{llllllll}
0 & 0 & 1 & 1 & 1 & 1 & 0 & 0 \\
0 & 1 & 0 & 1 & 1 & 0 & 1 & 0 \\
0 & 1 & 1 & 0 & 0 & 1 & 1 & 0
\end{array}\right] \\
C_{2} & =\left[\begin{array}{llllllll}
1 & 0 & 0 & 0 & 0 & 1 & 1 & 1 \\
1 & 0 & 0 & 0 & 0 & 1 & 1 & 1 \\
1 & 0 & 0 & 0 & 0 & 1 & 1 & 1
\end{array}\right] \\
C_{3} & =\left[\begin{array}{llllllll}
0 & 0 & 0 & 0 & 0 & 0 & 0 & 0 \\
0 & 0 & 0 & 0 & 0 & 0 & 0 & 0 \\
1 & 1 & 1 & 1 & 1 & 1 & 1 & 1
\end{array}\right]
\end{aligned}
$$

and the encoded codeword $C=(1011101111011101$ 00101101).

Table 2 illustrates the code design procedure for a number of codes with lengths up to 64. This Table includes the quasiperfect codes listed in [16], and provides all parameters that are required for the design of a GAC. For example, the optimum $(20,11,5)$ code [16] can be designed by choosing a $3 \times 7$ basic array with the $R_{1}=(7,4,3)$ row and $(3,2,2)$ column codes. The first additional code $C_{2}$, should consist of a $(7,2,3)$ row and $(3,1,3)$ column codes while the second additional code $C_{3}$, should have $x_{11}$ repeated in the last row.

Similar to the technique, introduced by Forney [8], the proposed technique can be implemented for the design of the $(16,8,8)$ Nordstrom-Robinson code [20]. This code can be obtained from the $(24,12,8)$ Golay code by simple deletion of the second row in the $C_{1}, C_{2}, C_{3}$ and

\begin{tabular}{|c|c|c|c|c|c|c|c|c|c|c|c|c|c|c|c|c|c|c|c|c|c|}
\hline \multicolumn{3}{|c|}{$\begin{array}{l}\text { Information vector } \\
\left(x_{9}, x_{10}, x_{11}\right)\end{array}$} & \multicolumn{8}{|c|}{ Encoded codeword } & \multicolumn{3}{|c|}{$\begin{array}{l}\text { Information vector } \\
\left(x_{9}, x_{10}, x_{11}\right)\end{array}$} & \multicolumn{8}{|c|}{ Encoded codeword } \\
\hline 0 & 0 & 0 & 0 & 0 & 0 & 0 & 0 & 0 & 0 & 0 & 1 & 0 & 0 & 1 & 1 & 1 & 0 & 0 & 1 & 0 & 0 \\
\hline 0 & 0 & 1 & 1 & 1 & 0 & 1 & 0 & 0 & 0 & 1 & 1 & 0 & 1 & 1 & 1 & 0 & 0 & 1 & 0 & 1 & 0 \\
\hline 0 & 1 & 0 & 1 & 0 & 1 & 1 & 0 & 0 & 1 & 0 & 1 & 1 & 0 & 0 & 1 & 0 & 1 & 0 & 1 & 1 & 0 \\
\hline 0 & 1 & 1 & 1 & 0 & 0 & 1 & 1 & 1 & 0 & 0 & 1 & 1 & 1 & 1 & 0 & 0 & 0 & 0 & 1 & 1 & 1 \\
\hline
\end{tabular}
C.

Table 1 : Code table of $(8,3)$ code

\subsection{Other known codes}

The technique described can be readily implemented for the design of other known codes. For example, following the procedure outlined above, one can design the $(32,6$, 16) $\mathrm{RM}$ code by choosing a $4 \times 8\left(n_{1}=4, n_{2}=8\right)$ basic array code, $C_{1}$, with an $R_{1}=(8,1,8)$ row and $(4,3,2)$ single parity check column codes. The first additional product code, $C_{2}$, should consist of a $4 \times 5$ matrix $P$ and a $4 \times 3$ matrix $A$ of repetition column codes. The third additional product code, $C_{3}$, must be an all zero matrix. The $(31,6,15)$ code can be derived easily be deleting the parity check symbol which is located in the 4th row and 8th column.

The $(32,26,4)$ and $(32,16,8) \mathrm{RM}$ codes which were discussed in [8] can also be designed by using the proposed algorithm. The $(31,26,3)$ and $(31,16,7)$ codes, which are equivalent to the corresponding $\mathrm{BCH}$ codes, can be derived from the corresponding RM codes by deleting the parity check symbols, located in the last column and row. It is apparent that since any $R M$ code can be decomposed into two codes with shorter code lengths [19] the concept of GACs can be applied for the design of all RM codes.

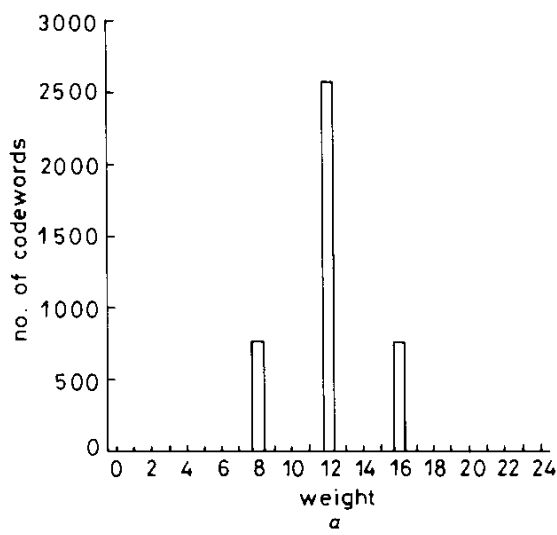

Fig. 4 Golay code weight distribution function

$a(24,12,8)$ Golay code $\quad b(23,12,7)$ Golay code

IEE Proc-Commun., Vol. I42, No. 4, August 1995

\section{Trellis design procedure}

The trellis design procedure for GACs [12] can be extended for the design of low-complexity regular trellises for all the codes described above. The procedure is as follows:

(i) Choose the trellis depth (number of columns), $N_{c}$, and number of states, $N_{s}$, as

$$
N_{c}=n_{2}=1 \quad N_{s}=2^{\max k_{p}}
$$

(ii) Identify each state of depth $p$ by the $\max \left\{k_{p}\right\}$-tuple binary vector $S_{p}(A)=S_{p}\left(a_{1}, a_{2}, \ldots, a_{\max \{k p\}}\right)$, where $a_{j}=$ 0,1 .

(iii) The trellis branches start and finish at depth $p=0$ and $p=n_{2}$; these are labelled as $S_{0}(00, \ldots, 0)$ and $S_{n 2}(00$, $\ldots, 0$ ), respectively.

(iv) The trellis branches at depth $p$ are labelled $X_{p} / C_{p}$, where $X_{p}$ represent $k_{p}$-tuple binary vectors of information digits for the $p$ th row code and $C_{p}$ correspond to the encoded codewords in the $p$ th row:

$$
C_{p}=X_{p}^{1} G_{p}+C_{p}^{2}
$$

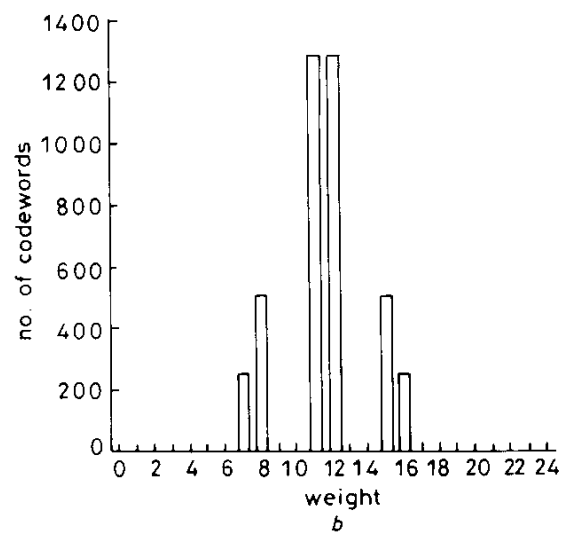


where $G_{p}$ is a generator matrix for the pth row code and $X_{p}^{1}$ and $C_{p}^{2}$ are codewords from the $p$ th rows of the $C_{1}$ and $C_{2}$ codes, respectively.

(v) There are $2^{k p}$ branches starting from each state, $S_{p}(A)$, at depth $p, p<N_{c}$; each branch is connected with state $S_{p+1}(A)$ at depth $p+1$, which is defined as follows:

$$
S_{p+1}\left(A_{j}\right)=S_{p}\left(A_{i}\right)+X_{p}
$$

(vi) If a second additional code, $C_{3}$, is used for a code design, at the final depth all states must be connected to final state, $S_{n}(00, \ldots, 0)$, with two parallel branches; the labels of these branches complement to each other.

There are

$$
N_{0}=\prod_{p=1}^{n 2} 2^{k_{p}}
$$

distinct paths through this trellis diagram and each path corresponds to a unique codeword from the code.

\subsection{Trellis diagrams of the $(8,4,4)$ and $(7,4,3)$ codes}

Let our aim be to design the trellis diagram of the $(8,4,4)$ code described in the Section 2.1. Following the technique outlined above, the trellis diagram of the $(8,4,4)$ code will have $N_{\mathrm{c}}=4+1=5$ columns and $N_{\mathrm{s}}=2^{2}=4$ states. We identify the states by a 2 -tuple binary vectors and at states $p=0$ and $p=5$ the trellis has only one state, namely $S_{0}(00)$ and $S_{4}(00)$, respectively. Following the above procedure, the trellis diagram for the $(8,4,4)$ code is presented in Fig. $5 a$, and is similar to that given by Forney $[8,9]$. The trellis diagram of the $(7,4,3)$ Hamming code is similar to the trellis of the $(8,4,4)$ code and differs only in the number of digits being used for labelling the branches at final depth (Fig. $5 b$ ).

3.2 Trellis diagrams of $(16,5,8)$ and $(15,5,7)$ code Now, let our aim be to design the trellis diagrams of the $(16,5,8)$ and $(15,5,7)$ code described in the Section 2.2 . Since the trellis diagram of the $(15,5,7)$ code can be derived from the trellis of the $(16,5,8) \mathrm{RM}$ code we start this example with the trellis design for $(16,5,8)$ code. The trellis will have $N_{c}=4+1=5$ columns and $N_{s}=2^{3}=8$ states. We identify each state by a 3-tuple binary vector and at depths $p=0$ and $p=4$, the trellis has only one state, namely $S_{0}(000)$ and $S_{4}(000)$, respectively. Following the above procedure, the trellis diagram for the $(16,5,8)$ GAC will be similar to that given by Forney for a corresponding $R M$ code [8]. The trellis diagram of the $(15,5,7)$ code can be derived easily by deleting one parity check symbol in the labelling of branches at depth $p=5$, and is presented in Fig. $6 b$. It is apparent that the designed codes have similar trellis diagrams, which differ only in the number of digits being used for labelling the branches

\begin{tabular}{|c|c|c|c|c|c|c|c|c|}
\hline No. & $\begin{array}{l}\text { Type of } \\
\text { code }\end{array}$ & $\mathbf{R}$ & $\begin{array}{l}\text { Size } \\
\text { of } \\
\text { array }\end{array}$ & $\begin{array}{l}\text { Row } \\
\text { code } \\
\text { in } C_{1}\end{array}$ & $\begin{array}{l}\text { Column } \\
\text { code in } \\
C_{1}\end{array}$ & $\begin{array}{l}\text { Row } \\
\text { code } \\
\text { in } C_{2}\end{array}$ & $\begin{array}{l}\text { Last } \\
\text { row } \\
\text { in } C_{3}\end{array}$ & $\begin{array}{l}\text { Last } \\
\text { symbol }\end{array}$ \\
\hline $\begin{array}{r}1 \\
2 \\
3 \\
4 \\
5 \\
6 \\
7 \\
8 \\
9 \\
10 \\
11 \\
12\end{array}$ & $\begin{array}{l}d_{\min }=3 \\
(6,3) \\
(7,4) \\
(9,5) \\
(9,6) \\
(11,6) \\
(12,8) \\
(13,7) \\
(15,11) \\
(18,12) \\
(30,20) \\
(45,30) \\
(63,42)\end{array}$ & $\begin{array}{l}0.5 \\
0.57 \\
0.55 \\
0.67 \\
0.545 \\
0.67 \\
0.538 \\
0.73 \\
0.67 \\
0.67 \\
0.67 \\
0.67\end{array}$ & $\begin{array}{l}2 \times 3 \\
2 \times 4 \\
2 \times 5 \\
3 \times 3 \\
2 \times 6 \\
3 \times 4 \\
2 \times 7 \\
4 \times 4 \\
3 \times 6 \\
3 \times 10 \\
3 \times 15 \\
3 \times 21\end{array}$ & $\begin{array}{l}(2,1,2) \\
(2,1,2) \\
(2,1,2) \\
(3,2,2) \\
(2,1,2) \\
(3,2,2) \\
(2,1,2) \\
(4,3,2) \\
(3,2,2) \\
(3,2,2) \\
(3,2,2) \\
(3,2,2)\end{array}$ & $\begin{array}{l}(3,2,2) \\
(4,3,2) \\
(5,4,2) \\
(3,2,2) \\
(6,5,2) \\
(4,3,2) \\
(7,6,2) \\
(4,3,2) \\
(6,5,2) \\
(10,9,2) \\
(15,14,2) \\
(21,20,2)\end{array}$ & $\begin{array}{l}(2,1,1) \\
(2,1,1) \\
(2,1,1) \\
(3,1,1) \\
(2,1,1) \\
(3,1,1) \\
(2,1,1) \\
(4,1,1) \\
(3,1,1) \\
(3,1,1) \\
(3,1,1) \\
(3,1,1)\end{array}$ & $\begin{array}{l}n / a \\
n / a \\
n / a \\
(3,1,3) \\
n / a \\
(3,1,3) \\
n / s \\
(4,1,4) \\
(3,1,3) \\
(3,1,3) \\
(3,1,3) \\
(3,1,3)\end{array}$ & $\begin{array}{l}\text { exists } \\
\text { deleted } \\
\text { deleted } \\
\text { exists } \\
\text { deleted } \\
\text { exists } \\
\text { deleted } \\
\text { deleted } \\
\text { exists } \\
\text { exists } \\
\text { exists } \\
\text { exists }\end{array}$ \\
\hline $\begin{array}{l}13 \\
14 \\
15 \\
16 \\
17 \\
18 \\
19\end{array}$ & $\begin{array}{l}d_{\min }=4 \\
(8,4) \\
(10,5) \\
(12,7) \\
(14,7) \\
(15,9) \\
(16,11) \\
(30,19)\end{array}$ & $\begin{array}{l}0.5 \\
0.5 \\
0.58 \\
0.5 \\
0.6 \\
0.69 \\
0.63\end{array}$ & $\begin{array}{l}2 \times 4 \\
2 \times 5 \\
3 \times 4 \\
2 \times 7 \\
3 \times 5 \\
4 \times 4 \\
3 \times 10\end{array}$ & $\begin{array}{l}(2,1,2) \\
(2,1,2) \\
(3,2,2) \\
(2,1,2) \\
(3,2,2) \\
(4,3,2) \\
(3,2,2)\end{array}$ & $\begin{array}{l}(4,3,2) \\
(5,4,2) \\
(4,3,2) \\
(7,6,2) \\
(5,4,2) \\
(4,3,2) \\
(3,2,2)\end{array}$ & $\begin{array}{l}(2,1,1) \\
(2,1,1) \\
(3,1,1) \\
(2,1,1) \\
(3,1,1) \\
(4,1,1) \\
(3,1,1)\end{array}$ & $\begin{array}{l}n / a \\
n / a \\
n / a \\
n / a \\
n / a \\
(4,1,4) \\
n / a\end{array}$ & $\begin{array}{l}\text { exists } \\
\text { exists } \\
\text { exists } \\
\text { exists } \\
\text { exists } \\
\text { exists } \\
\text { exists }\end{array}$ \\
\hline $\begin{array}{l}20 \\
21 \\
22 \\
23 \\
24\end{array}$ & $\begin{array}{l}d_{\min }=5 \\
(15,7) \\
(17,9)) \\
(19,10) \\
(20,11) \\
(21,12)\end{array}$ & $\begin{array}{l}0.47 \\
0.53 \\
0.53 \\
0.55 \\
0.57\end{array}$ & $\begin{array}{l}5 \times 3 \\
6 \times 3 \\
7 \times 3 \\
7 \times 3 \\
7 \times 3\end{array}$ & $\begin{array}{l}(5,2,3) \\
(6,3,3) \\
(7,4,3) \\
(7,4,3) \\
(7,4,3)\end{array}$ & $\begin{array}{l}(3,2,2) \\
(3,2,2) \\
(3,2,2) \\
(3,2,2) \\
(3,2,2)\end{array}$ & $\begin{array}{l}(5,2,2) \\
(6,2,2) \\
(7,1,3) \\
(7,2,3) \\
(7,3,3)\end{array}$ & $\begin{array}{l}(5,1,5) \\
(6,1,6) \\
(5,1,5) \\
(6,1,6) \\
(6,1,6)\end{array}$ & $\begin{array}{l}\text { exists } \\
\text { deleted } \\
2 \text { deleted } \\
\text { deleted } \\
\text { exists }\end{array}$ \\
\hline $\begin{array}{l}25 \\
26 \\
27\end{array}$ & $\begin{array}{l}d_{\min }=7 \\
(15,5) \\
(23,12) \\
(31,16) \\
(63,32)\end{array}$ & $\begin{array}{l}0.33 \\
0.52 \\
0.52 \\
0.51\end{array}$ & $\begin{array}{l}4 \times 4 \\
8 \times 3 \\
8 \times 4 \\
8 \times 8\end{array}$ & $\begin{array}{l}(4,1,4) \\
(8,4,4) \\
(8,4,4) \\
(8,4,4)\end{array}$ & $\begin{array}{l}(3,2,2) \\
(3,2,2) \\
(4,3,2) \\
(8,7,2)\end{array}$ & $\begin{array}{l}(4,2,2) \\
(8,3,3) \\
(8,3,3) \\
(8,3,3)\end{array}$ & $\begin{array}{l}\mathrm{n} / \mathrm{a} \\
(7,1,7) \\
(7,1,7\rangle \\
(7,1,7)\end{array}$ & $\begin{array}{l}\text { deleted } \\
\text { deleted } \\
\text { deleted } \\
\text { deleted }\end{array}$ \\
\hline $\begin{array}{l}29 \\
30 \\
31\end{array}$ & $\begin{array}{l}a_{\min }^{\prime}=8 \\
(16,5) \\
(24,12) \\
(32,16) \\
(64,32)\end{array}$ & $\begin{array}{l}0.31 \\
0.5 \\
0.5 \\
0.5\end{array}$ & $\begin{array}{l}4 \times 4 \\
8 \times 3 \\
8 \times 4 \\
8 \times 8\end{array}$ & $\begin{array}{l}(4,1,4) \\
(8,4,4) \\
(8,4,4) \\
(8,4,4)\end{array}$ & $\begin{array}{l}(3,2,2) \\
(3,2,2) \\
(4,3,2) \\
(8,7,2)\end{array}$ & $\begin{array}{l}(4,2,2) \\
(8,3,3) \\
(8,3,3) \\
(8,3,3)\end{array}$ & $\begin{array}{l}n / a \\
(8,1,8) \\
(8,1,8) \\
(8,1,8)\end{array}$ & $\begin{array}{l}\text { exists } \\
\text { exists } \\
\text { exists } \\
\text { exists }\end{array}$ \\
\hline
\end{tabular}
at the greatest depth. 


\subsection{Trellis diagrams of the $(16,11,4)$ and $(15,11,3)$} codes

The trellis diagram of the $(16,11,4) \mathrm{GAC}$ can be designed in two different ways [12]:

(i) with $N_{c}=5$ columns and $N_{c}=16$ states (if no parallel branches are used);

(ii) with $N_{c}=5$ columns and $N_{s}=8$ states (if parallel branches are used at every depth of the trellis).

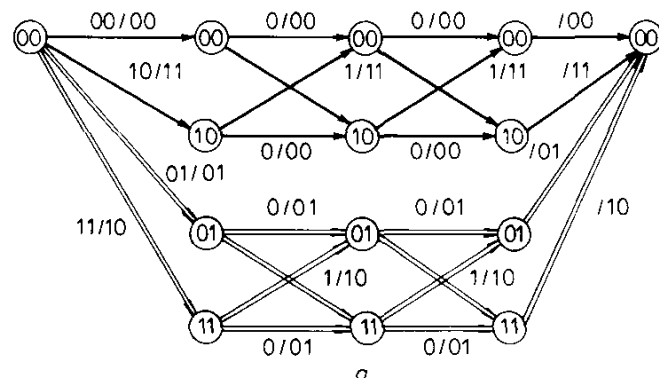

Fig. 5 Trellis diagrams

$a(8,4,4)$ RM code $b(7,4,3)$ Hamming code

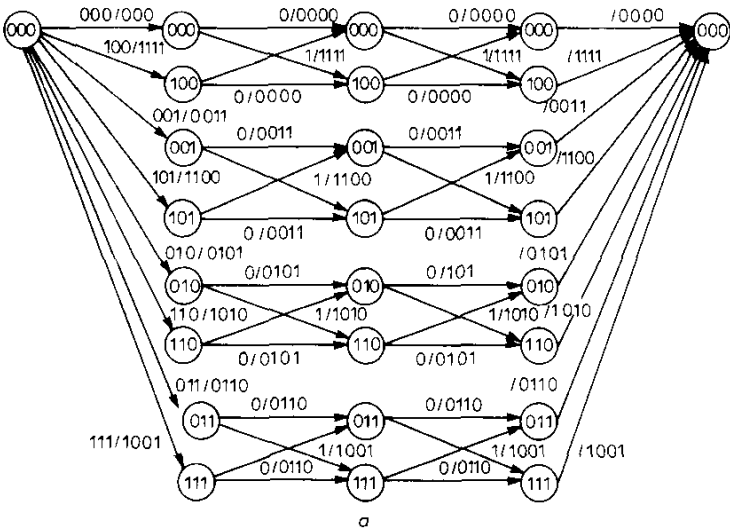

Fig. 6 Trellis diagrams

$a(16,5,8)$ RM code $b(15,5,7) \mathrm{GAC}$

In both cases, the trellis has $2^{11}$ different paths and is isomorphic to the trellis diagram of the $(16,11,4) \mathbf{R M}$ code [8]. The $(15,11,3)$ Hamming code will have a similar trellis with the only difference being that three digits are used for labelling of branches at the final depth.

\subsection{Trellis diagram of the $(15,7,5)$ code}

The proposed technique can be applied to the design of the trellis diagram for the $(15,7,5)$ code described previously. Following the procedure outlined above, the trellis will have $N_{s}=16$ and $N_{c}=4$. Each state of the trellis is identified by a 4-tuple binary vector and trellis branches are labelled according to eqn. 26. Since a second additional code, $C_{3}$, has been used for the code design, at the depth $p=N_{c}$ each state is connected to the state $S_{3}(0000)$ with two parallel branches, as is shown in Fig. 7. The number of states for the designed $(15,7,5)$ code can be reduced to 8 , if parallel branches are used at each depth of the trellis diagram [18]

\subsection{Trellises of other known codes}

The above technique can be implemented for the trellis design of almost all known codes. Figs 8-11 present trellis diagrams for different codes which were obtained by using the new technique. The trellis diagram of the
$(31,6,15)$ (Fig. 8$)$ has the following parameters: $N_{s}=16$, $N_{c}=5$ and is similar to the trellis diagram of the $(32,6$, 16) $R M$ code [8]. The only difference is in the number of digits which are used for labelling branches in the last depth of the trellis.

The trellis diagram of the $(24,12,8)$ Golay code (Fig. 9) represents a set of 8 similar subtrellises. Each subtrellis has 8 states which are connected by the two parallel
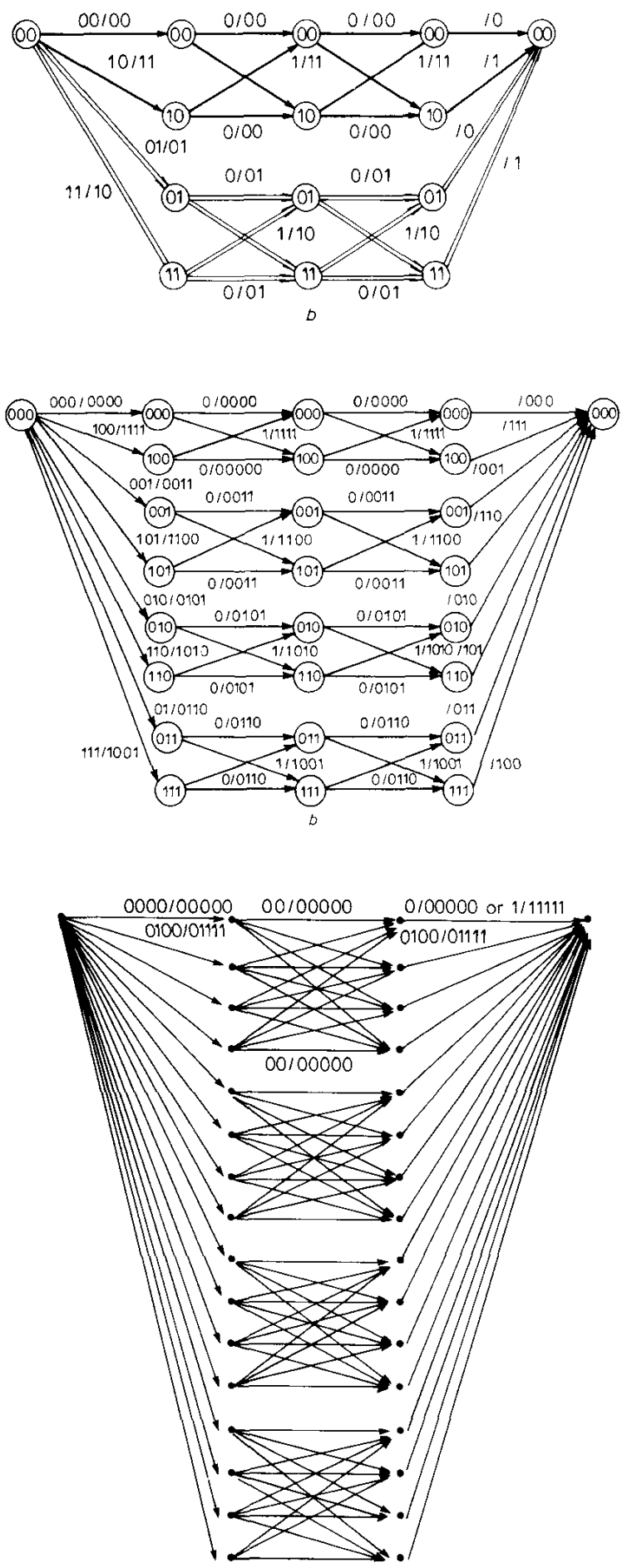

Fig. 7 Trellis diagram of $(15,7,5) G A C$ 
branches. The labelling of each subtrellis can be easily obtained from the labelling of the first subtrellis by adding corresponding codewords of the $(8,3,3)$ code (Table 1). The overall trellis has the following parameters: $N_{s}=64, N_{c}=3$ and is similar to the trellis diagram given in Reference 8 . The trellis diagram of the $(23,12,7)$ code has the same structure as $(24,12,8)$ code

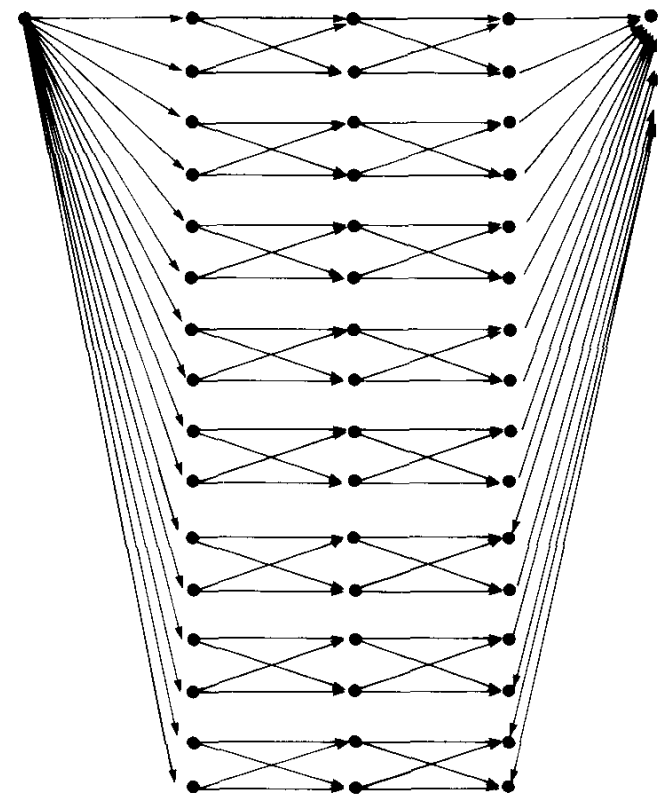

It is apparent from these Figures that the code design technique described in this paper provides codes with regular trellis structure, thus allowing the low-complexity trellis decoding techniques, such as coset decoding, to be implemented.

\section{Conclusion}

A new low-complexity encoding technique for linear block codes, based on generalised array codes, is

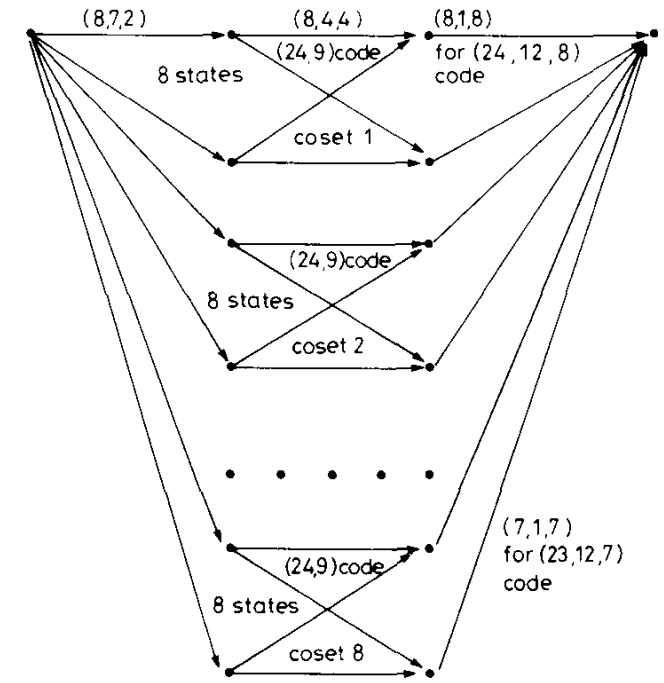

Fig. 9 Trellis diagrams of Golay codes

Fig. 8 Trellis diagram of $(31,6,15)$ code

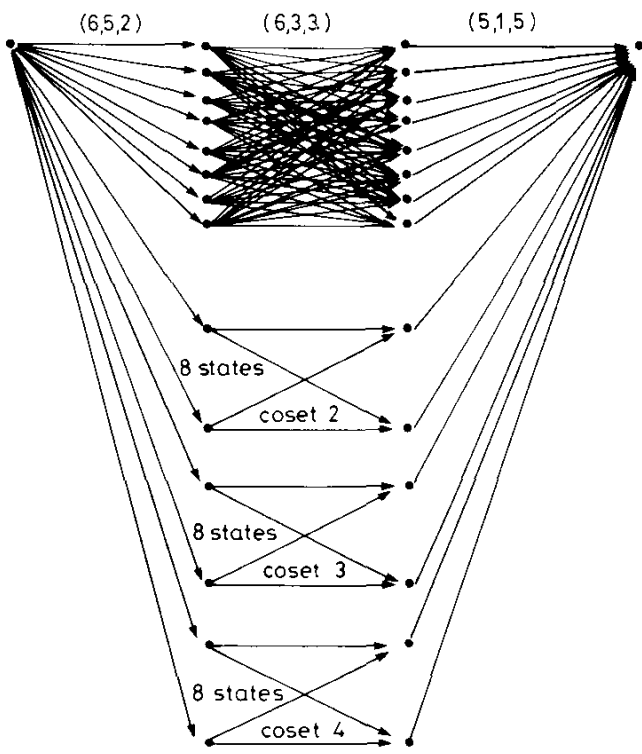

Fig. 10 Trellis diagram of $(17,9,5)$ GAC

and differs only in number of digits which are used for labelling branches in the last column of the trellis (all branches in this column are labelled with 7-tuple codewords).

The trellis diagram of the $(17,9,5)$ code has the following parameters: $N_{s}=32, N_{\mathrm{c}}=3$, and is shown in Fig. 10. The trellis diagrams for some quasi-perfect codes are shown in Fig. 11. described. The technique allows the design of linear block codes together with their low-complexity trellises. Examples are presented for a number of widely used codes, (Reed-Muller, Hamming, Golay, optimum etc.); however, the technique can be employed in the design of a wide variety of known linear block codes. The designed trellises have a regular structure and allow low-complexity VLSI and DSP implementations. 

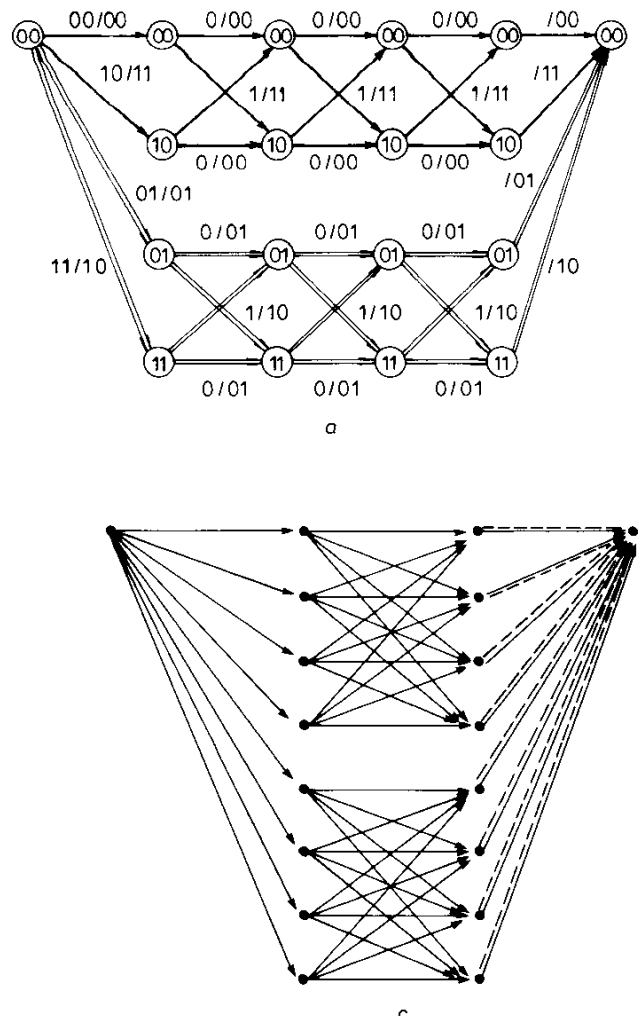

Fig. 11 Trellis diagrams of $G A C s$

$a(10,5,4) \mathrm{GAC} \quad b(14,7,4) \mathrm{GAC} \quad c(9,6,3) \mathrm{GAC} \quad d(12,8,3) \mathrm{GAC}$

\section{References}

1 BAHL, L.R., COCKE, J., JELINEK, F., and RAVIV, J.: 'Optimal decoding of linear codes for minimising symbol error rate', $I E E E$ Trans. Inf. Theory, 1974,20 , pp. 284-287

2 WOLF, J.K.: 'Efficient maximum likelihood decoding of linear block codes using a trellis', IEEE Trans. Inf. Theory, 1978, 24, (1), pp. $76-80$

3 MASSEY, J.: 'Foundation and methods of channel encoding'. Proceedings of international conference on Information theory, 65, 18-20 Sept., 1978, Berlin

4 CONWAY, J.H., and SLOANE, N.J.: 'Soft decoding techniques for codes and lattices, including Golay code and the Leech lattice', IEEE Trans. Inf. Theory, 1986, 32, (1), pp. 41-50

5 KASAMI, T., TAKATA, T., FUJIWARA, T., and LIN, S.: 'On complexity of trellis structure of linear block codes', IEEE Trans. Inf. Theory, 1993, 39, (1), pp. 242-245

6 SNYDERS, J.: 'Reduced lists of error patterns for maximum likelihood soft decoding, IEEE Trans. Inf. Theory, 1991, 37, (4), pp. 667 672

7 BE'ERY, Y., and SNYDERS, J.: 'Optimal soft decision decoders based on fast Haddamard transform', IEEE Trans. Inf. Theory, 1986, 32, pp. 355-364

8 FORNEY, G. D.: 'Coset codes - Part 2: Binary lattices and related codes', IEEE Trans. Inf. Theory, 1988, 34, (5), pp. 1152-1187

9 FORNEY, G.D., and TROTT, M.D.: 'The dynamics of group codes: state spaces, trellis diagrams, and cannonical encoders', IEEE Trans. Inf. Theory, 1993, 39, (5), pp. 1491-1523
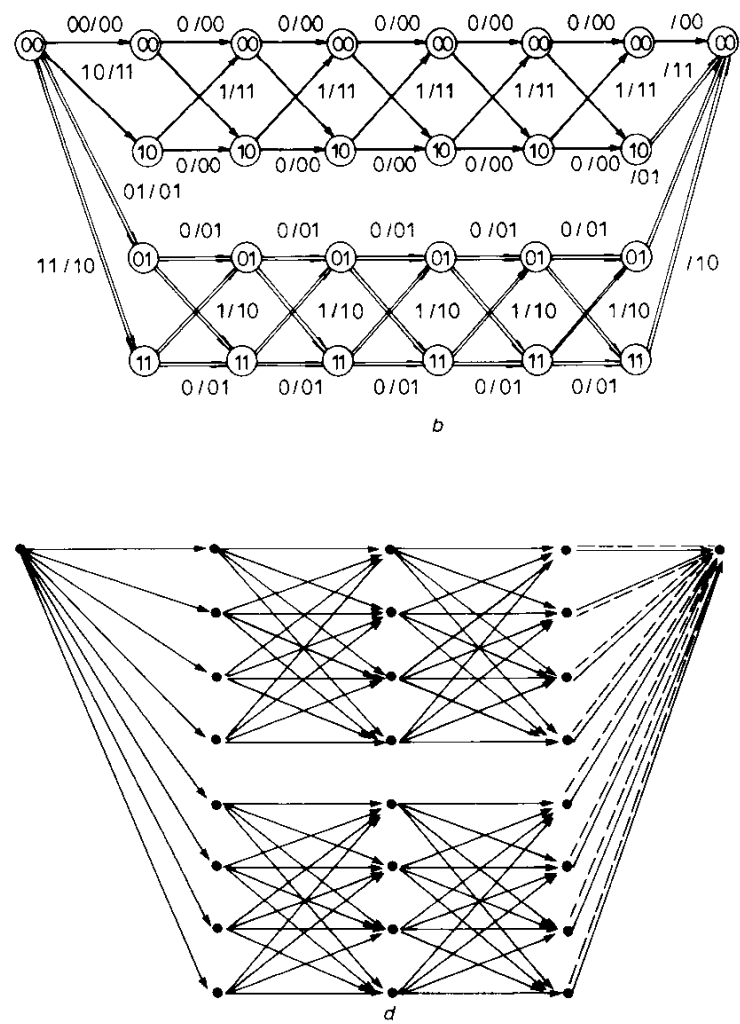

10 MUDER, D.J.: 'Minimal trellises for block codes', IEEE Trans. Inf. Theory, 1988, 34, (5), pp. 1049-1053

11 BERGER, Y, and BE'ERY, Y.: 'Bounds of the trellis size of linear block codes', IEEE Trans. Inf. Theory, 1993, 39, (1), pp. 203-208

12 HONARY, B., MARKARIAN, G., and FARRELL, P.: 'Generalised array codes and their trellis structure'. Electron. Lett., 1993, 29, (6), pp. 541-542

13 HONARY, B., and MARKARIAN, G.: 'Low complexity trellis decoding of Hamming codes', Electron. Lett, 1993, 29, (12), pp. 1114-1116

14 HONARY, B., and MARKARIAN, G.: 'New simple encoder and trellis decoder for Golay codes', Electron. Lett., 1993, 29, (25), pp. 2170-2171

15 LIN, S., and COSTELLO, D.: 'Error control coding: fundamental and applications' (Prentice-Hall, Englewood Cliffs, NJ, 1983)

16 PETERSON, W.W., and WELDON, E.J.: 'Error correcting codes' (MIT Press, 2nd edn., 1975)

17 GOETHALS, J.M.: 'On the Golay perfect binary code', J. Combinatorial Theory, 1971, 11, pp. 178-186

18 ZYABLOV, V.V., and SIDORENKO, V.: 'Bounds of complexity of trellis decoding of linear blockcodes'. Proceedings of the SwedishRussian workshop on Information theory, Aug. 1993, Sweden

19 MCWILLIAMS, F.J., and SLOANE, N.J.A.: 'The theory of error correcting codes'. NHPC, 1978.

20 NORDSTROM, A.W., and ROBINSON, J.P.: 'An optimum nonlinear code', Inf. \& Control, 1967, 11, pp. 613-616 